\title{
Primary non-Hodgkin diffuse large B-cell lymphoma of the lacrimal sac: a rare case of aggressive tumor and literature review
}

\author{
Maria Gabriella Malzone ${ }^{1}$, Marzio Di Meglio ${ }^{2}$, Dario Furgiuele ${ }^{2}$, \\ Nicola Galantuomo ${ }^{3}$, Sabrina Alfano ${ }^{1}$, Gennaro Mossetti ${ }^{1}$
}

1) Pathological Anatomy Service, Maria Rosaria Clinic, Pompeii, Naples, Italy

2) Department of Ophthalmology, Maria Rosaria Clinic, Pompeii, Naples, Italy

3) Laservista Ophthalmology center, Caserta, Italy
DOI: $10.15386 / \mathrm{mpr}-1926$

Manuscript received: 03.10 .2020

Received in revised form: 10.12 .2020

Accepted: 25.12.2020

Address for correspondence:

gabriellamalzone@gmail.com

This work is licensed under a Creative Commons Attribution-NonCommercialNoDerivatives 4.0 International License

\begin{abstract}
Primary malignant lymphomas of the lacrimal sac are rare neoplasms. They are mainly B-cell lymphoma with nonspecific and atypical symptoms, which can lead to misdiagnosis and a delay in the appropriate cure. Early diagnosis made by biopsy and the right choice of treatments can increase the chance of survival. We report a case of a non-Hodgkin diffuse large B-cell lymphoma (DLBCL) of the lacrimal sac, involving also the drainage system, in a 77-year-old female. She presented with an epiphora, swelling and a heavy visual impairment at the left eye successfully treated with cycles of polychemotherapy and radiotherapy.
\end{abstract}

Keywords: non-Hodgkin diffuse large B-cell lymphoma, lacrimal sac, epiphora, polychemotherapy

\section{Introduction}

Lymphoproliferative diseases of the orbit represent $10-15 \%$ of all ocular tumors [1]. They can originate in the periorbital and/or orbital region (primary lymphoma) or be the result of a systemic lymphoreticular spread (secondary lymphoma). A careful histomorphological and immunophenotypical examination is required for the classification of more than 40 different subtypes of lymphoma, all with different behavior and prognosis [2-5]. Clinical signs and symptoms are nonspecific and they vary based on the area of the eye affected [6].

Primary non-Hodgkin's lymphoma (NHL) of the lacrimal sac is extremely rare $[7,8]$.

According to the World Health Organization (WHO) classification of all primary lacrimal sac tumors, diffuse large B-cell lymphoma (DLBCL) occurs at $43 \%$; it is known to be aggressive but potentially curable $[9,10]$, with clinicopathological features and optimal management not well defined. Patients can be managed with multimodality treatments: surgical excision is often the first approach for decompression of swelling and proptosis followed by either chemotherapy or radiation, or even a combination of both, depending on the lymphoma subtype, age and general status of the patient.

Herein, we provide a rare case of a 77-year-old female with a primary ocular DLBCL of the lacrimal sac. She successfully underwent partial surgical excision of the lesion followed by cycles of polychemotherapy and radiotherapy, showing a remarkable improvement with a shrinkage of the tumor already from the first chemotherapy cycle.

\section{Case report}

In January 2020, a 77-year-old woman affected by diabetes mellitus and hypertensive heart disease, presented to her ophthalmologist with an epiphora and a secretion upon awakening for about three months, also complaining of a heavy visual impairment in the left eye over the previous year. On physical examination, the ophthalmologist detected an epiphora and mild serum-mucous reflux of the left lacrimal sac region, where a wellcircumscribed swelling was also evidenced, 
hard and painless. The lower lacrimal ducts were occluded to washing. She started a topical and systemic antibiotic therapy, for approximately one month, without any benefit but with a significant proptosis (Figure 1A). Therefore, the patient was referred to an oculoplastic surgeon for advice, who confirmed the previous diagnosis and added a corticosteroid therapy, in anticipation of the intervention. After 2 days of therapy with $25 \mathrm{mg}$ deltacortene, the swelling appeared to be slightly regressive, but the therapy had to be suspended due to failure of the diabetic pathology.

At the end of February, the patient was admitted to our facility to undergo pre-operative examinations. A facial
CT scan revealed the presence of a large neoformation that invaded the deep left orbit, deformed and dislocated the bulb and reached the front of the eyelid and the root of the ipsilateral nose (Figure 1B). The bone structures and the lymph nodes stations of the head and neck district were intact. One week later a trans-cutaneous orbitotomy type surgery with an arched incision at the inferior internal orbital edge was approached: a tissue of lardaceous color and consistency was found which diffusely infiltrated the surrounded tissues. Therefore, numerous biopsy specimens were taken and a secondary stenosis of the lacrimal ducts was noted, which appeared not infiltrated.
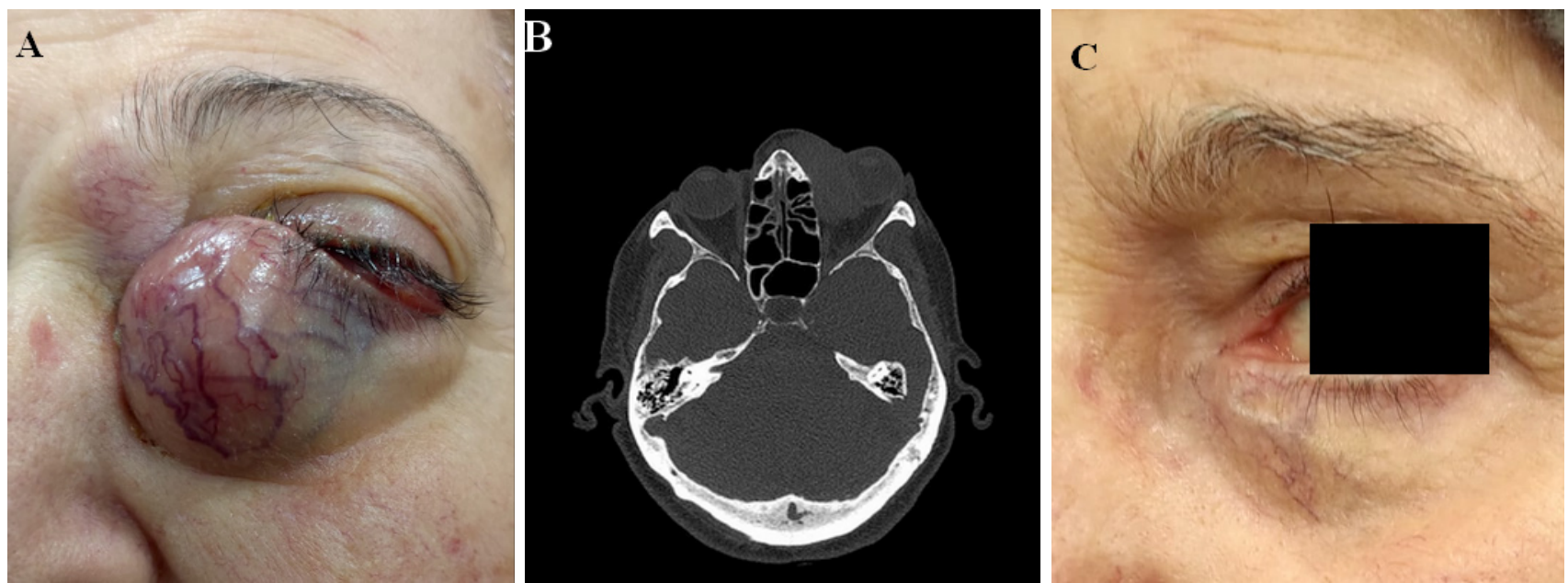

Figure 1 A. 77-year-old patient with significant proptosis of the left eye before surgery; B. CT scan showing the lacrimal sac tumor of the left orbit before surgery and chemotherapy; C. Complete resorption of the lacrimal sac tumor after surgery and the first cycle of polychemotherapy.
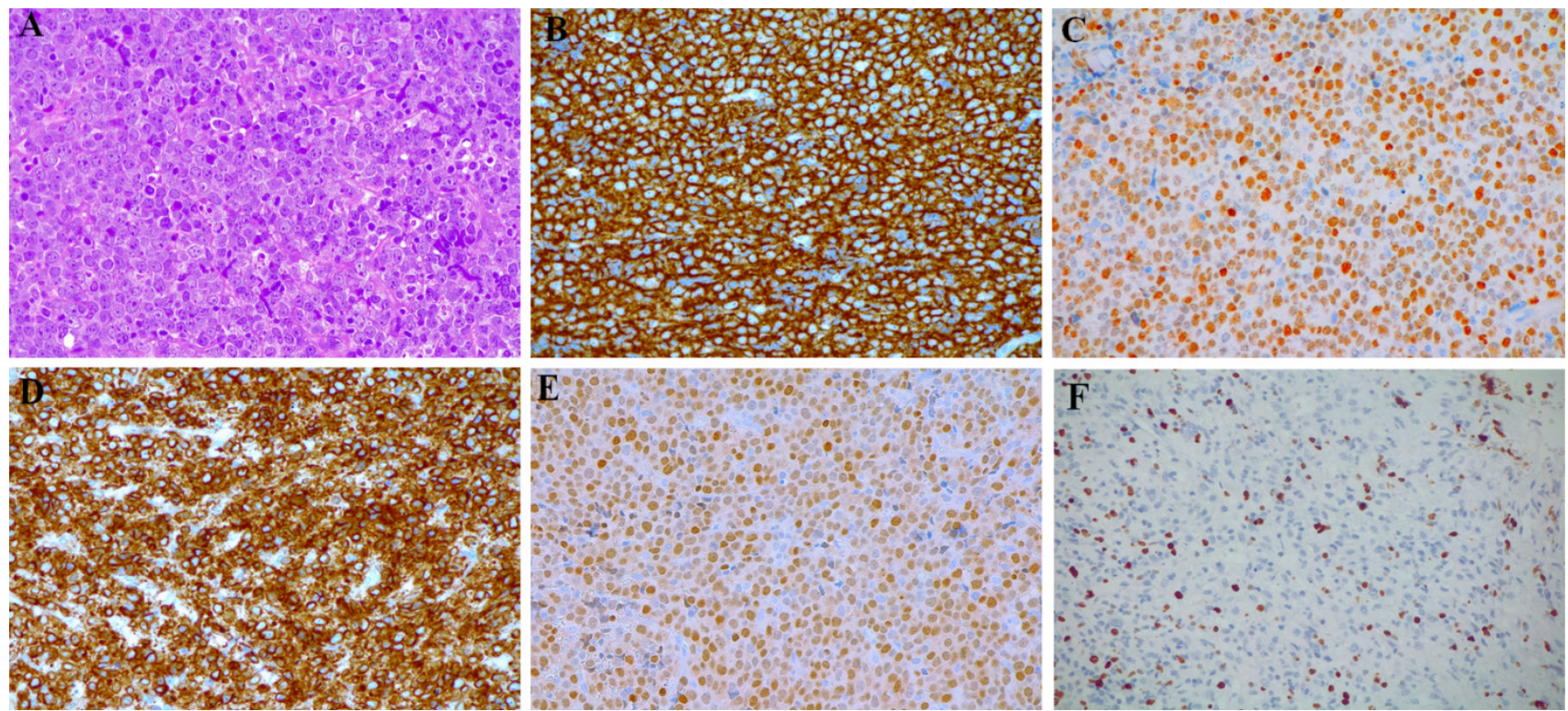

Figure 2 A. Surgical sample of the orbital area. The histomorphological picture is consistent with non-Hodgkin lymphoma, diffuse large $\mathrm{B}$-cell variant, non-germinal center phenotype according to Hans algorithm (H and E, X200, original magnification). Immunohistochemistry staining was positive for CD20 (B); BCL-6 (C); BCL-2 (D); MUM-1 (E); C-MYC (20\%) (F) (X200, original magnification). 
Histopathological analysis of the paraffin embedded tissues revealed a stromal tissue extensively infiltrated by a lymphoid proliferation, with a diffuse growth pattern, consisting of large B-cells (CD20+) characterized by eosinophilic cytoplasm, large nuclei with dispersed chromatin and evident nucleoli. Mitotic figures were highly frequent. Immunohistochemical stains showed positivity to BCL-6, BCL-2, MUM-1, C-MYC and negativity to CD3, CD5, CD10, CD23, ALK-1 and Cyclin D-1 (Figure $2 \mathrm{~A}-\mathrm{F}$ ). $\mathrm{Ki}-67$ index was greater than $90 \%$. The final diagnosis was of non-Hodgkin lymphoma, diffuse large B-cell variant, non-germinal center phenotype according to Hans algorithm [11].

In March, the patient was referred to the Operational Unit of Oncological Hematology to complete tumor staging. During hospitalization she underwent a bone marrow biopsy and a Positron Emission Tomography (PET) total body, which were both negative. The patient was nominated for a program that included 2 cycles of polychemotherapy, every 28 days, according to the R-COMP scheme by Luminari and collaborators [12] with the addition of Methotrexate (3000 mg/m $/ \mathrm{m}^{2}$ g 5 in 3 hours), plus 4 cycles of R-COMP. She showed a marked improvement already from the first chemotherapy cycle with a shrinkage of the tumor (Figure 1C); the only side effect observed was a painful stomatitis, which affected the whole mouth. In November, the latest PET total body revealed no evidence of systemic lymphoma but only a small localized tumefaction at the left lacrimal sac region. Therefore, 1 cycle of radiotherapy for consolidation was planned.

\section{Discussion}

Primary lymphoma of the lacrimal sac is exeptionally rare, comprising approximately $1 \%$ of nonHodgkin lymphomas and $8 \%$ of extranodal lymphomas $[13,14]$ with most cases being of B-cell type and secondary to metastatic lymphoproliferative disease [15].

DLBCL is a typical high-grade tumor, with an aggressive behavior but not impossible to cure. As reported in several studies, DLBCL could represent the transformation/progression of MALT lymphoma, a less aggressive type of lymphoma. The possible delay in the lacrimal sac diagnosis, due to its rather hidden location, can increase the chance of extra genetic alterations inducing a transformation from MALT lymphoma to DLBCL $[16,17]$. Diagnosis of DLBCL can be difficult due to atypical and nonspecific symptoms at presentation, which often lead to misdiagnosis of dacryocystitis [18]. The common predominant features of lacrimal sac lymphoma are epiphora and painless swelling of the lacrimal sac area, which can be both associated with dacryocystitis. Our patient presented a heavy visual impairment in the left eye, epiphora with a secretion and a well-circumscribed hard and painless swelling of the lacrimal sac region, followed by a significant proptosis.
Computed Tomography (CT) or Magnetic Resonance Imaging (MRI) are initially used not only to evaluate the location, the extension and the possible systemic lymphomatous involvement, but also to plan the most appropriate surgical approach and subsequent treatment [19]. Our patient underwent a pre-operative facial CT scan, which showed the presence of a large neoformation, evenly enhanced on contrast, which deeply invaded the left orbit, deformed the bulb and reached the root of the ipsilateral nose, leaving the remaining bone structures intact. Biopsy is always required for definitive diagnosis of lacrimal sac tumors $[2,20]$. The histological analyses of the transcutaneous orbitotomy of our patient that referred neither a history of a previous lymphoma nor had any manifestations of systemic lymphoma, showed a non-Hodgkin large B-cell lymphoma, non-germinal center phenotype, confirmed by immunohistochemical staining. Petrella and collaborators in a Phase III trial demonstrated that BCL2 expression but not MYC and BCL2 co-expression in non-germinal center B-cell-like phenotype lymphoma, appeared to confer a worse progression-free survival and overall survival [21].

No common treatment guidelines exist for orbital lymphoma management: the choice depends on the histological type, localization, extension of the disease, age and disease-related prognostic factors. A multimodality approach including surgery, chemotherapy and/or radiotherapy is successfully implied. It is general opinion that total surgical resection of orbital lymphoma is to be avoided for the risk of damage to the eye with consequent partial or total loss of vision. Surgery should be used just for diagnostic purposes [22,23].

One of the main limitations in the treatment of aggressive NHL in elderly and unfit patients is the cardiotoxicity of anthracyclines, which however in this type of lymphomas exert their greatest therapeutic efficacy. Therefore, various polychemotherapy schemes have been proposed with conflicting results [24,25]. Recently a nonpegylated liposomal doxorubicin (Myocet), a drug that has a more favorable cardiotoxicity profile, has been used. Studies conducted on elderly and unfit patients have shown that the R-COMP regimen (analogous to R-CHOP with the replacement of Myocet $50 \mathrm{mg} / \mathrm{m}^{2}$ instead of doxorubicin) determines complete response rates of $57-79 \%$ and an overall survival of $70-77 \%[12,26]$. In these studies, serious adverse events of a cardiac nature were absent or involved a very low percentage of patients, indicating that liposomal doxorubicin allows to maintain adequate therapeutic efficacy with fewer side effects. In our patient R-COMP scheme was combined with intravenous Methotrexate to further improve complete remission, overall survival and reduce relapses without serious adverse reactions $[27,28]$.

Until the end of November 2020 the disease of our patient remained localized and she appeared in general good health. 


\section{Conclusion}

Early diagnosis of primay DLBCL of the lacrimal sac is difficult to make due to nonspecific symptoms at presentation. Our patient had been suffering, for about 3 months, of epiphora, secretion and swelling of the lacrimal sac area, which can be considered as early predictive symptoms of malignant lymphomas, but they can be also associated with acute purulent dacryocystitis and mucoceles. Besides, the heavy visual impairment experienced by the patient over the previous year was not a specific sign for neoplasia, as she suffered from severe cataracts. Only the combination of these symptoms, with sudden proptosis and the use of imaging, led to a strong suspicion of neoplasia and immediate surgery.

Primay DLBCL can have a diverse clinical course, with an overall survival rate of $36 \%$ [29], but the choice of multimodality treatments after appropriate staging can successfully reduce recurrence and progression of the disease and increase the 3-year overall survival rate up to $77 \%$ [12]. At nine months follow-up the patient is doing well, with only a small localized tumefaction. After the cycle of radiotherapy, she hopes to undergo cataract removal surgery.

\section{References}

1. Knowles DM 2nd, Jakobiec FA. Ocular adnexal lymphoid neoplasms: clinical, histopathologic, electron microscopic, and immunologic characteristics. Hum Pathol. 1982;13:148162.

2. Alkatan HM, Alaraj AM, Al-Ayoubi A. Diffuse large B-cell lymphoma of the orbit: A tertiary eye care center experience in Saudi Arabia. Saudi J Ophthalmol. 2012;26:235-239.

3. Coupland SE, Krause L, Delecluse HJ, Anagnostopoulos I, Foss HD, Hummel M, et al. Lymphoproliferative lesions of the ocular adnexa. Analysis of 112 cases. Ophthalmology. 1998;105:1430-1441.

4. Coupland SE, Hellmich M, Auw-Haedrich C, Lee WR, Stein $\mathrm{H}$. Prognostic value of cell-cycle markers in ocular adnexal lymphoma: an assessment of 230 cases. Graefes Arch Clin Exp Ophthalmol. 2004;242:130-145.

5. Sriram PR. A Rare Case of Aggressive, Huge Primary Orbital Lymphoma with Intracranial Extension and Bone Invasion. Asian J Neurosurg. 2017;12:766-768.

6. Specht CS. Benign and malignant lymphoid tumors, leukemia and histiocytic lesions. In: Principles and practice of ophthalmology (eds. by D.M. Albert, F.A. Jakobiec). WB Saunders Philadelphia. 1994; pp. 3328-3350.

7. Tőrők-Vistai T, Bojan A, Cucuianu A, Zsoldos A. Primary non-Hodgkin lymphoma of the orbit presenting with massive bilateral periorbital tumors. Clujul Med. 2013;86:380-382.

8. Sjö LD, Ralfkiaer E, Juhl BR, Prause JU, Kivelä T, AuwHaedrich $\mathrm{C}$, et al. Primary lymphoma of the lacrimal sac: an EORTC ophthalmic oncology task force study. $\mathrm{Br} \mathrm{J}$ Ophthalmol. 2006;90:1004-1009.
9. Singh S, Ali MJ. Lymphoproliferative tumors involving the lacrimal drainage system: a major review. Orbit. 2020;39:276-284.

10. Swerdlow SH, Campo E, Harris NL, Jaffe ES, Pileri SA, Stein $\mathrm{H}$, et al. WHO classification of tumors of haematopoietic and lymphoid tissues. Revised 4th Edition, Volume 2. IARC Press. Lyons. 2017; pp. 291-297.

11. Hans CP, Weisenburger DD, Greiner TC, Gascoyne RD, Delabie J, Ott G, et al. Confirmation of the molecular classification of diffuse large B-cell lymphoma by immunohistochemistry using a tissue microarray. Blood. 2004;103:275-282.

12. Luminari S, Montanini A, Caballero D, Bologna S, Notter M, Dyer MJS, et al. Nonpegylated liposomal doxorubicin (Myocet TM) combination (R-COMP) chemotherapy in elderly patients with diffuse large B- cell lymphoma (DLBCL): results from the phase II EUR018 trial. Ann Oncol. 2009;21:1492-1499.

13. Pelloski CE, Wilder RB, Ha CS, Hess MA, Cabanillas FF, Cox JD. Clinical stage IEA-IIEA orbital lymphomas: outcomes in the era of modern staging and treatment. Radiother Oncol. 2001;59:145-151.

14. Stefanovic A, Lossos IS. Extranodal marginal zone lymphoma of the ocular adnexa. Blood. 2009;114:501-510.

15. Stefanyszyn MA, Hidayat AA, Pe'er JJ, Flanagan JC. Lacrimal sac tumors. Ophthalmic Plast Reconstr Sur. 1994;10:169-184.

16. Moslehi R, Devesa SS, Schairer C, Fraumeni JF Jr. Rapidly increasing incidence of ocular non-hodgkin lymphoma. J Natl Cancer Inst. 2006;98:936-939.

17. Matolcsy A. High-grade transformation of low-grade nonHodgkin's lymphomas: mechanisms of tumor progression. Leuk Lymphoma. 1999;34:251-259.

18. Palamar M, Midilli R, Ozsan N, Egrilmez S, Sahin F, Yagci A. Primary diffuse large B-cell lymphoma of the lacrimal sac simulating chronic dacryocystitis. Auris Nasus Larynx. 2011;38:643-645.

19. Khan SN, Sepahdari AR. Orbital masses: CT and MRI of common vascular lesions, benign tumors, and malignancies. Saudi J Ophthalmol. 2012;26:373-383.

20. Schalenbourg A, Mantel I. The eye and cancer. Rev Med Suisse. 2015;11:2395-2398.

21. Petrella T, Copie-Bergman C, Brière J, Delarue R, Jardin F, Ruminy P, et al. BCL2 expression but not MYC and BCL2 coexpression predicts survival in elderly patients with diffuse large B-cell lymphoma independently of cell of origin in the phase 3 LNH03-6B trial. Ann Oncol. 2017;28:1042-1049.

22. Venkitaraman R, George MK. Primary non Hodgkin's lymphoma of the lacrimal sac. World J Surg Oncol. 2007;5:127.

23. Esik O, Ikeda H, Mukai K, Kaneko A. A retrospective analysis of different modalities for treatment of primary orbital nonHodgkin's lymphomas. Radiother Oncol. 1996;38:13-18.

24. Thieblemont C, Bernard S, Molina T. Management of aggressive lymphoma in very elderly patients. Hematol Oncol. 2017;35(Suppl Suppl 1):49-53. 
25. Merli F, Bertini M, Luminari S, Mozzana R, Botto B, Liberati AM, et al. Long term results of a randomized study performed by Intergruppo Italiano Linfomi comparing MiniCEOP vs P-VEBEC in elderly patients with diffuse large B-cell lymphoma. Leuk Lymphoma. 2007;48:367-373.

26. Gimeno E, Sánchez-González B, Alvarez-Larrán A, Pedro C, Abella E, Comín J, et al. Intermediate dose of nonpegylated liposomal doxorubicin combination (R-CMyOP) as first line chemotherapy for frail elderly patients with aggressive lymphoma. Leuk Res. 2011;35:358-362.

27. Boccomini C, Ladetto M, Rigacci L, Arcaini L, Ceccarelli M, Lobetti-Bodoni C, et al. Brief chemoimmunotherapy
Rituximab, Bendamustine, Mitoxantrone (R-BM) followed by Rituximab consolidation in elderly patients with untreated advanced stage follicular lymphoma (FL): preliminary results of a prospective phase II study by Fondazione Italiana Linfomi (FIL). Blood. 2012;120:Abstract 2720.

28. Frenkel S, Hendler K, Siegal T, Shalom E, Pe'er J. Intravitreal methotrexate for treating vitreoretinal lymphoma: 10 years of experience. Br J Ophthalmol. 2008;92:383-388.

29. Munch-Petersen HD, Rasmussen PK, Coupland SE, Esmaeli B, Finger PT, Graue GF, et al. Ocular adnexal diffuse large B-cell lymphoma: a multicenter international study. JAMA Ophthalmol. 2015;133:165-73. 